\title{
What Is Next for Retinal Gene Therapy?
}

\author{
Luk H. Vandenberghe \\ Ocular Genomics Institute, Grousbeck Gene Therapy Center, Schepens Eye Research Institute, \\ Massachusetts Eye and Ear Infirmary, Harvard Medical School, Boston, Massachusetts 02114 \\ Correspondence: luk_vandenberghe@meei.harvard.edu
}

The field of gene therapy for retinal blinding disorders is experiencing incredible momentum, justified by hopeful results in early stage clinical trials for inherited retinal degenerations. The premise of the use of the gene as a drug has come a long way, and may have found its niche in the treatment of retinal disease. Indeed, with only limited treatment options available for retinal indications, gene therapy has been proven feasible, safe, and effective and may lead to durable effects following a single injection. Here, we aim at putting into context the promise and potential, the technical, clinical, and economic boundaries limiting its application and development, and speculate on a future in which gene therapy is an integral component of ophthalmic clinical care.

\section{PROMISE DELIVERED}

Three hree critical components have to be defined and brought together for any therapeutic approach to be successful: target, intervention, and delivery. The target requires the identification of a cell type, tissue, or process relevant to the pathophysiology of the disease. The information on these targets can then be used to devise an intervention through which a disease process can be inhibited, circumvented, or interfered with. The bundling of this intervention with an approach to deliver it to the target within a therapeutic window in a manner that is feasible, safe, and efficient constitutes an attractive treatment paradigm. Over the past two or three decades, efforts of the vision and neuroscience research community have converged with those of the genetics and gene delivery field to lead to the definition of targets, the evalua- tion of multiple intervention modalities, and the validation of a host of delivery systems.

The first incarnation of this approach was targeting a form of Leber congenital amaurosis (LCA), an early onset form of autosomal recessive retinal degeneration leading to progressive vision loss and nystagmus. RPE65, a gene encoding an enzyme pivotal in the recycling of the visual pigment chromophore 11-cis-retinal, was identified as one of the genes leading to LCA when mutated on both alleles (Marlhens et al. 1997). The addition of a correct copy of the $R P E 65$ gene led to the restoration of gene expression in this loss-of-function, single gene disorder (Acland et al. 2001). In parallel, the field of in vivo gene transfer was embarking on evaluating technologies for therapeutic applications, out of which the adeno-associated viral vector (AAV) emerged as a minimally immunogenic vector capable of stably transducing nondividing cells

Editors: Eric A. Pierce, Richard H. Masland, and Joan W. Miller

Additional Perspectives on Retinal Disorders: Genetic Approaches to Diagnosis and Treatment available at

www.perspectivesinmedicine.org

Copyright (C) 2015 Cold Spring Harbor Laboratory Press; all rights reserved; doi: 10.1101/cshperspect.a017442

Cite this article as Cold Spring Harb Perspect Med 2015;5:a017442 
L.H. Vandenberghe

(Ali et al. 1996). An unconventional surgical route of delivery that layered the gene therapy vector below the retina, adjacent to the therapeutic target cell type, that is, the retinal pigment epithelium (RPE), was proven to be essential for allowing AAV to come to its full potential in terms of gene transfer efficiency, specificity, and safety (Bennett et al. 1994; Ali et al. 1996). Bundled, these pivotal findings led to a demonstration, in a canine model of the disease, that subretinal injection of AAV encoding a promoter driven cDNA of RPE65 restored objective and behavioral measures of vision (Acland et al. 2001). Validated by three independent groups, clinical trials along this premise yielded promising results with moderate improvements in visual function of patients (Bainbridge et al. 2008; Cideciyan et al. 2008, 2009; Maguire et al. 2008). A successful phase 2 trial (Bennett et al. 2012) justified initiation of a currently active phase 3 study which is aimed at licensure of the first effective gene therapy drug in the United States.

The cumulative research in this area that led to this sequence of events in less than two decades is a model in this era of translational medicine, and has generated an increased interest in gene therapy applications to target ophthalmic disease, particularly for indications in which no treatment options are available or current therapeutic paradigms are inadequate or suboptimal. Moreover, these studies disrupted a prevalent school of thought that experimental treatments such as gene therapy could only be applied in fatal disorders for which no therapies were available.

\section{CARBON COPY?}

In 2012, The National Institutes of Health Office of Biotechnological Activities convened several stakeholders in gene therapy. At this meeting, it was suggested that future retinal degeneration therapies could be developed in a streamlined fashion by building on a delivery platform established in the RPE65 studies (O'Reilly et al. 2013). The idea is to use an identical vector type, surgical procedure, and transgene cassette to deliver therapeutic genes for other retinal indi- cations. Indeed, the example that these studies have set, provides a guide for the design and development of gene therapies for other forms of retinal blindness.

The need for streamlining the development of these innovative therapies is high. Treatment options for many retinal blinding disorders are limited to nonexisting yet numerous therapeutic approaches and targets are being pursued preclinically. This requires a multitude of therapies to be developed, for example, there are more than 200 genes causative of inherited retinal disorders when mutated (see https://sph.uth.edu/ RetNet/sum-dis.htm\#A-genes). Moreover, a variety of different interventions are considered, ranging from gene addition, neuroprotection, and optogenetics. This, added to the targets and intervening modalities in more complex retinal disease, such as age-related macular degeneration (AMD), amounts to a large translational need (Sahel and Roska 2013; Simonato et al. 2013). The time and cost of translating a preclinical proof-of-concept that brings together target, intervention, and delivery is extremely high, and any cost-saving or streamlining of this process would bring transformative therapies to the patient faster.

Since the publication, in 2008, of the results of the early phase studies on the RPE65 gene therapy trials, extensive efforts have been underway to build on this momentum, and to deliver a second success built on this same mold. In 2014, promising data from a phase $1 / 2$ clinical trial for gene augmentation therapy for choroideremia, an X-linked form of retinal degeneration caused by defects in the REP-1 protein, was presented (MacLaren et al. 2014). These studies used the same vector (AAV2), delivery route (subretinal injection), and promoter (cytomegalovirus [CMV] enhanced chicken $\beta$-actin) as two of the RPE65 studies, evidently building on, and adding to, the platform potential of this approach. It is important to highlight also the point of divergence in design of the gene therapy, and its translational path to clinical studies. First, certain design elements of the transgene were added, most notably the woodchuck hepatitis virus posttranscriptional response element (WPRE) known to increase 
What is Next for Retinal Gene Therapy?

mRNA half-life and thereby lead to enhanced transgene expression. Second, the targeted area for treatment extended, much more than in the RPE65 studies into the macula, the area of the retina where in these patients' most target cells remained viable substrates for gene therapy. In terms of the body of evidence supporting the clinical translation for this second gene target, it appeared less exhaustive animal data was sufficient (Tolmachova et al. 2013). This is an important evolution as animal models of the retinal dystrophies are often lacking, not reflecting human disease, or only demonstrating a slow progressing or mild phenotype within the animal's lifespan (Chang et al. 2002). Also, to take advantage of the clinical validation AAV2 received in the prior studies, improved AAV serotypes that are thought to lead to a more robust expression in photoreceptor cells, next to RPE targets for the gene transfer (Boye et al. 2012; Vandenberghe and Auricchio 2012; Vandenberghe et al. 2011), were not pursued here, possibly limiting the efficacy potential of the study. This highlights an important decision point in the pursuit of developing a novel retinal gene therapy: Does one take advantage of a platform such as AAV2, risking subtherapeutic levels of expression in important target cells, or does one diverge from the validated platform at the risk of complicating the translational path of dealing with more unknowns and higher regulatory hurdles?

\section{A PIPELINE OF PROGRESS}

A cadre of clinical studies is currently ongoing, pending, or actively being developed at a preclinical stage (Boye et al. 2013). Many of these studies build on AAV2, but often also chose for a different vector including alternative AAV serotypes or lentiviral systems, either for improved targeting, or to overcome the limited transgene size capacity of AAV. Once results emerge from the clinic, these alternative vector systems may become novel platforms that can be built on. Specifically, trials are ongoing using a lentiviral vector encoding ABCA4 and MYO7A for the treatment of Stargardt disease (Binley et al. 2013) and Usher Type 1B (Zallocchi et al.
2014), respectively. These cDNAs are too large to be packaged in $\mathrm{AAV}$, yet can be accommodated by a lentiviral vector. A concern with this vector system has been the low transduction efficiency for photoreceptor cells, a primary therapeutic target for both these diseases (Auricchio et al. 2001; Bainbridge et al. 2001; Binley et al. 2013). Two clinical studies are underway with a gene therapy approach for durable expression of an antiangiogenic molecule in the treatment of exudative AMD (Maclachlan et al. 2011; Lai et al. 2012). Interestingly, the two trials use similar therapeutic molecules (sFlt1) and AAV2 as a vector, however differ in the surgical delivery of the gene therapy; a group out of Lions Eye Institute in Australia in collaboration with Avalanche Biosciences injects subretinally (Lai et al. 2012), whereas Genzyme/Sanofi is pursuing intravitreal injection (Maclachlan et al. 2011). The outcome of these approaches will inform us on another gene therapy modality: gene transfer for sustained delivery of a secreted therapeutic protein drug. Several gene augmentations approaches are underway clinically: all are AAV2-based, one building on the RPE65 results but directed at MERTK (NCT01482195), a disease-causing gene in retinitis pigmentosa, and another set of studies for Leber hereditary optic neuropathy (LHON) caused by mutations in the mitochondrial gene ND4 (NCT02161380 and NCT02064569), which is expressed in the retinal ganglion cells that make up the optic nerve. Dozens of other preclinical programs are at various stages of development, for example, AAV5 mediated expression of GUCY2D for treatment of another form of LCA, which is soon expected to head to the clinic (Boye et al. 2013).

In short, following a pioneering era of firstin-human, a number of studies are bound to yield results, some building and solidifying the subretinal AAV2 approach, whereas others hopefully will expand the toolset and therapeutic reach for retinal degeneration gene therapies.

\section{IMPROVING ON EFFICACY}

In more than a dozen gene therapy clinical trials of several hundreds of subjects, safety endpoints 
have been met in the absence of significant, drug-related adverse events (Simonato et al. 2013). No phase 3 studies have been completed, and efficacy in a randomized controlled trial has yet to be established for any of the ongoing approaches, although these results are anticipated in the near future for a phase 3 study for one of the RPE65 gene therapies. Reports on efficacy in retinal gene therapy, therefore, have been limited to the phase 1/2 RPE65 and choroideremia (CHM) studies. Data presented there has been promising and demonstrating increased light sensitivity by a variety of measures, and in some subjects improved navigation of a mobility course, indicating not only biological effect of the treatment, but also the potential for clinical benefit. However, based on the remarkable disease rescue observed in dog models following AAV2.RPE65 gene therapy, which appears not as robust as in humans, several hypotheses have been suggested to explain the apparent differences in treatment effect.

Cideciyan and colleagues (2013a) followed treated subjects and compared those to the natural disease progression in dogs and humans as well as the therapeutic effects seen in dogs over time. Using spectral domain optical coherence tomography (SD-OCT) and a novel methodology to model the decay of retinal structure, the investigators concluded that in humans, as opposed to canines, retinal degeneration starts earlier, and the optimal intervention for a gene therapy in this form of LCA is likely before birth (Cideciyan et al. 2013a). Their data suggests treated subjects show stable visual function in the context of ongoing retinal degeneration. To improve both visual function and structural preservation, the investigators propose a combination therapy in which neuroprotective treatment is combined with the gene addition. These results have extensively been argued, over its unorthodox modeling, the limited longitudinal data from patients (as opposed to dogs) following treatment, the small data set with several variables that are unaccounted for in the analysis, and its suggested solution to overcome this concern (Cepko and Vandenberghe 2013; Cideciyan et al. 2013b; Townes-Anderson 2013; Wojno et al. 2013). Moreover, only data from one of the clinical studies was included, making it difficult to assess if the observations can be extended to the other trial designs.

Alternative hypotheses argue that the limitations of the current gene therapy formulations and surgical approaches can be improved on by targeting a larger area of the retina than a subretinal injection can reach, the level of transgene expression is limiting for full treatment effect, or the specificity of expression and stoichiometry of RPE65 in the context of the visual cycle is not in balance, arguing for more specific and regulated expression. These interventions may not have been required in the murine or canine models because of species-specific parameters such as the size of the globe, the procedural difference in terms of surgery, molecular kinetics of vector uptake, transduction, or promoter activity that are possibly distinct in canine versus human retina.

These discussions highlight, in our view, the need to continue to define the therapeutic window for each approach and indication, and to seek to improve the technology and biology of the treatment. Clinical, ethical, and pragmatic boundaries limit us in considering prenatal or neonatal (and before some of these studies, pediatric) gene therapy in ophthalmology, which, at least for now, determines the start of the potential therapeutic window for intervention. The field is in agreement that there is also a clear closing of the window when the therapeutic target cell (often RPE and/or photoreceptors) are atrophied. What is also agreed on, however less defined, is the threshold at which for many of these progressively degenerative diseases, degeneration cannot be stemmed in a cell autonomous fashion. Elucidation and diagnosis of that transition point is essential for identifying the appropriate therapeutic approach for each patient. Technological improvement to gene therapy that can restore gene function in a more physiological manner is likely also to prove important. The prospect of genome editing therapy in this respect is a fascinating one, because this technology may permit the adoption of endogenous regulation of gene expression. Albeit early, and faced with critical hurdles still, the recent advances in this field make this 
approach compelling (Cong et al. 2013; Mali et al. 2013).

While this debate and detailed level of analysis is essential to progress, we cannot lose track of the fact that the noted clinical studies have concluded a biological effect in the absence of harm, with the potential of remarkable, clinically relevant, improvements of visual function. Absent of other treatment options for these patients, this first generation gene therapy is a milestone that can be used as a benchmark for future improvements. It is also important to note that these improvements often can only be validated in cautious, yet pivotal, human clinical studies. The careful translation of this experimental paradigm that has led to the first demonstration of efficacy in the context of safety for in vivo gene therapy is therefore a salute to the need for experimental clinical research to move these important questions forward.

\section{WIDENING THE SPECTRUM}

The compelling data from the early clinical trials, and the pipeline of preclinical studies moving to translation, has illustrated, for many, the transformative potential of gene therapy in ophthalmic care. It needs to be noted, however, that the current studies only address very small patient populations, and, at least in the short to mid-term, the prospect of gene therapy alleviating blinding disorders on a wider scale is limited. Several parameters determine this current limitation: (1) LCA, owing to mutations in RPE65 and CHM, only affect few, and many other forms of inherited retinal degeneration will require a different treatment; (2) more common disorders, such as AMD, are currently only in clinical trials in populations at the very late stage of the disease, and even when proven safe and effective in current trials it remains to be seen how these results can be extended to a wider wet AMD population; (3) the therapeutic window for most of the approaches currently in trials is limited to those with remaining target cells and some level of vision; and (4) the prohibitive cost of clinical translation and trial costs for experimental biological therapies.
To broaden the potential of gene therapy for wider patient populations in ophthalmology, progress is made on several fronts. Not unlike the development of this first generation of therapies, the convergence of several fields including genetics, gene delivery, retinal biology, neuroscience, and cell therapy, is leading the charge in this effort. New targets are continuously being identified through gene discovery and studies into the pathophysiology of retinal disease; new disease genes in inherited retinal degenerations are being discovered, as are new targets in AMD and other common-complex retinal disorders. Novel and improved paradigms to intervene are now also more actively considered for vision restoration, in part, because of the traction and excitement spurred by the retinal gene therapy trial success. Specifically, the restoration of vision through gene transfer of an optogenetic switch that is accurately embedded within the neural circuitry of the retina is pursued by several groups and may lead to some level of visual perception in the blind, even in the absence of (endogenous) photoreceptors (Busskamp et al. 2012). Advances in neuroprotection through survival factors, antiapoptotic, or antioxidant agents will be beneficial to other gene therapy strategies, or may be delivered in a sustained fashion via gene transfer (Sahel and Roska 2013). The intersect of gene transfer and genome editing with cell-based transplantation approaches is highly promising to enable visual restoration even when retinal degeneration is extensive, and more traditional approaches of gene therapy are outside of consideration. Finally, progress in the field of gene delivery can further unlock further applications. The limitation on the size of the transgene imposed by the clinically used vector systems currently is not only preventing gene addition in the most common forms of LCA and RP (CEP290 and USH2A, respectively), it also often prevents transgene cassettes to be designed with transcriptional or translational elements (e.g., cellspecific promoters or introns) that would be beneficial, or multiple genes to be transferred in a single vector (e.g., gene replacement combined with a neuroprotective gene). Novel vector technologies like nanoparticle approaches 
L.H. Vandenberghe

(Conley and Naash 2010), or methods like genome editing to restore endogenous gene regulation, are expected to have an important impact. To further expand on the paradigm pursued by the pending AMD trials delivering an antiangiogenic protein by gene therapy to achieve long-lived and stable drug delivery to overcome repeat injections in a chronic disease, novel and improved methods to modulate transgene expression will become essential. Somewhat surprisingly, although several methods are available (Zoltick and Wilson 2001), few of them are actively pursued clinically in a gene therapy context, in part due to vector size limitations and the complexity related to translating these systems to human use. Last, efficient and safe targeting from the vitreous would overcome several shortcomings of the subretinal injection route and, certainly, make gene therapy a more routine clinical procedure (Dalkara et al. 2013; Kay et al. 2013).

\section{MEASURING OUTCOME}

Shared with other experimental therapeutic strategies in ophthalmology, the ability to measure outcome is pivotal for gene therapy to become a clinical modality in the care of patients. This is primarily of importance to convincingly establish the level of efficacy these therapies bring. Equally important is the quest for early endpoints to assess therapeutic effect to shorten the "bench to bedside and back" life-cycle of the gene therapy development of these cost-heavy trials in often slowly progressing diseases. Because of the advantages that the visual system has in terms of access, diagnosis, and imaging, the measures to establish a biological effect of a treatment are available, and several of those have been used by the different groups pursuing ophthalmic gene therapy clinically. Moving forward, to the extent possible, a standardization of these measures will be beneficial to accurately compare outcomes from different trials and the variables between those. A higher bar to meet is the demonstration of clinical benefit, in part, because of the fact that a sensitive measure of biological effect may not equate to an improvement in quality of life or clinical status. Another aspect, however, is the regulatory definition of clinical benefit in ophthalmology that traditionally has been quite narrow. Because of the onslaught of novel therapeutic approaches for indications for which no treatments options are in current clinical use, disease organizations and clinical researchers continue a dialogue with regulators to establish new endpoint measures for clinical research, and ultimately market approval (Cellular, Tissue and Gene Therapies Advisory Committee 2011, see http://www.fda .gov/downloads/advisor.../ucm259087.pdf). While traditionally, visual acuity, and in limited cases visual field, has been acceptable, the RPE65 phase 3 study currently in progress uses a validated mobility assay as an endpoint (NCT 00999609). Validated surrogate measures such as those obtained via SD-OCT will likely also prove to be extremely valuable (Birch et al. 2013). The availability of a functionally validated biomarker to assess visual function, the stage of a disease process, or the activity of a therapeutic target is generally considered a "Holy Grail" for expediting and quantitation of therapeutic effect in humans. A key requirement for all endpoints, particularly in light of the fact that placebo controlled studies will remain difficult to design in gene therapy, is the availability of natural history studies to outline the disease course by that measure for that particular indication.

\section{ECONOMIC FACTORS}

With clinical proof-of-concept established, a delivery platform proposed, and a range of therapeutic targets and intervention of promise in the scientific literature, it may seem surprising and disappointing, particularly to patients and their families, that not more retinal gene therapies reached the clinic. As outlined above, several scientific, biological, and clinical limitations are at cause here. However, economic factors arguably play a primary role. The development of a single gene therapy is a timeand resource-demanding effort. Few centers have all the components under one roof to carry these efforts, and traditionally these have been pursued in academia with, until recently limited, interest from industry. The infrastructure 
What is Next for Retinal Gene Therapy?

cost to establish a preclinical and particularly a translational gene therapy center is extremely high, and in a declining funding environment, difficult to justify by academic institutions. In the past two years, by the excitement that the first clinical results have generated, fortunately, significant private investment has entered the arena (see http://www.forbes.com/sites/ matthewherper/2014/03/26/once-seen-as-tooscary-editing-peoples-genes-with-viruses-makesa-618-million-comeback/). To sustain this momentum, novel regulatory and economic frameworks are being developed that are tailored to this novel therapeutic domain. One important aspect here is the pricing of a gene therapy drug once on the market, which is expected to determine the incentive for industry to pursue development of these therapies (Brennan and Wilson 2014). Particularly, for gene-specific therapies in rare disorders, these aspects will become vital for continued development as therapies with a larger market are likely more desirable to investors. This is ironic as this field was founded and catapulted to success by demonstrating its potential for very rare disorders. To alleviate this tension, policy and regulatory changes have made orphan drug development more attractive, including extended patent protection, and distinct regulatory paths. The idea to work toward a platform that through clinical experience gains a higher regulatory comfort level is an attractive one, as it is expected to lead to a reduced cost and time of development. To meet this ambition, standardization has to be sought for in vector manufacturing and quality control, clinical trial design, endpoint measures, and clinical follow up. While this is a challenge in an increasingly competitive arena, we argue it is beneficial for both academic and private pursuits in terms of cost-savings and the rate at which therapies can be brought to the patients.

\section{A BRIGHT FUTURE}

Gene addition therapy in two forms of inherited retinal degenerations has proven to improve visual function, in some subjects with remarkable success, in the context of a relatively safe procedure and formulation. These results have validated an approach and a technology in a manner that it has the potential to alter the treatment of retinal disorders in the future. Although many aspects in this endeavor remain the subject of research, debate, policy and regulatory adjustments, a light at the end of the tunnel is a driving force for many in the field to pursue, mature, and create novel therapies for vision loss based on this paradigm. The extent to which gene therapy can broaden its impact beyond its current niche applications hinges on scientific and clinical advances, continued funding and investment, and balanced effort between competition and standardization of this promising field.

\section{ACKNOWLEDGMENTS}

We thank Livia Carvalho, Heikki Turunen, and Eric Pierce for discussion and manuscript comments. Support for this work was from National Institutes of Health (NIH) Grant DP1OD008267, Curing Kids Fund, Foundation for Retina Research, Foundation Fighting Blindness, Research to Prevent Blindness, and the Grousbeck Family Foundation. LHV is an inventor on patents related to AAV gene therapy; has served as a consultant and is inventor on technologies licensed to biotechnology and pharmaceutical industry; and is cofounder and consultant to GenSight Biologics.

\section{REFERENCES}

Acland GM, Aguirre GD, Ray J, Zhang Q, Aleman TS, Cideciyan AV, Pearce-Kelling SE, Anand V, Zeng Y, Maguire AM, et al. 2001. Gene therapy restores vision in a canine model of childhood blindness. Nat Genet 28: 92-95.

Ali RR, Reichel MB, Thrasher AJ, Levinsky RJ, Kinnon C, Kanuga N, Hunt DM, Bhattacharya SS. 1996. Gene transfer into the mouse retina mediated by an adeno-associated viral vector. Hum Mol Genet 5: 591-594.

Auricchio A, Kobinger G, Anand V, Hildinger M, O’Connor E, Maguire AM, Wilson JM, Bennett J. 2001. Exchange of surface proteins impacts on viral vector cellular specificity and transduction characteristics: The retina as a model. Hum Mol Genet 10: 3075-3081.

Bainbridge JW, Stephens C, Parsley K, Demaison C, Halfyard A, Thrasher AJ, Ali RR. 2001. In vivo gene transfer to the mouse eye using an HIV-based lentiviral vector; efficient long-term transduction of corneal endothelium 


\section{L.H. Vandenberghe}

and retinal pigment epithelium. Gene Ther 8: 16651668 .

Bainbridge JW, Smith AJ, Barker SS, Robbie S, Henderson R, Balaggan K, Viswanathan A, Holder GE, Stockman A, Tyler N, et al. 2008. Effect of gene therapy on visual function in Leber's congenital amaurosis. N Engl J Med 358: 2231-2239.

Bennett J, Wilson J, Sun D, Forbes B, Maguire A. 1994 Adenovirus vector-mediated in vivo gene transfer into adult murine retina. Invest Ophthalmol Vis Sci 35: 2535-2542.

Bennett J, Ashtari M, Wellman J, Marshall KA, Cyckowski LL, Chung DC, McCague S, Pierce EA, Chen Y, Bennicelli JL, et al. 2012. AAV2 gene therapy readministration in three adults with congenital blindness. Sci Transl Med 4: $120 \mathrm{ra} 115$.

Binley K, Widdowson P, Loader J, Kelleher M, Iqball S, Ferrige G, de Belin J, Carlucci M, Angell-Manning D, Hurst F, et al. 2013. Transduction of photoreceptors with equine infectious anemia virus lentiviral vectors: Safety and biodistribution of StarGen for Stargardt disease. Invest Ophthalmol Vis Sci 54: 4061-4071.

Birch DG, Locke KG, Wen Y, Locke KI, Hoffman DR, Hood DC. 2013. Spectral-domain optical coherence tomography measures of outer segment layer progression in patients with X-linked retinitis pigmentosa. JAMA ophthalmol 131: 1143-1150.

Boye SE, Alexander JJ, Boye SL, Witherspoon CD, Sandefer KJ, Conlon TJ, Erger K, Sun J, Ryals R, Chiodo VA, et al. 2012. The human rhodopsin kinase promoter in an AAV5 vector confers rod- and cone-specific expression in the primate retina. Hum Gene Ther 23: 1101-1115.

Boye SE, Boye SL, Lewin AS, Hauswirth WWA. 2013. A comprehensive review of retinal gene therapy. Mol Ther 21: 509-519.

Brennan TA, Wilson JM. 2014. The special case of gene therapy pricing. Nat Biotechnol 32: 874-876.

Busskamp V, Picaud S, Sahel JA, Roska B. 2012. Optogenetic therapy for retinitis pigmentosa. Gene Ther 19: 169-175.

Cepko CL, Vandenberghe LH. 2013. Retinal gene therapy coming of age. Hum Gene Ther 24: 242-244.

Chang B, Hawes NL, Hurd RE, Davisson MT, Nusinowitz S, Heckenlively JR. 2002. Retinal degeneration mutants in the mouse. Vision Res 42: 517-525.

Cideciyan AV, Aleman TS, Boye SL, Schwartz SB, Kaushal S, Roman AJ, Pang JJ, Sumaroka A, Windsor EA, Wilson JM, et al. 2008. Human gene therapy for RPE65 isomerase deficiency activates the retinoid cycle of vision but with slow rod kinetics. Proc Natl Acad Sci 105: 1511215117.

Cideciyan AV, Hauswirth WW, Aleman TS, Kaushal S, Schwartz SB, Boye SL, Windsor EA, Conlon TJ, Sumaroka A, Roman AJ, et al. 2009. Vision 1 year after gene therapy for Leber's congenital amaurosis. $N$ Engl J Med 361: 725-727.

Cideciyan AV, Jacobson SG, Beltran WA, Sumaroka A, Swider M, Iwabe S, Roman AJ, Olivares MB, Schwartz SB, Komáromy AM, et al. 2013a. Human retinal gene therapy for Leber congenital amaurosis shows advancing retinal degeneration despite enduring visual improvement. Proc Natl Acad Sci 110: E517-E525.
Cideciyan AV, Jacobson SG, Beltran WA, Hauswirth WW, Aguirre GD. 2013b. Reply to Townes-Anderson. RPE65 gene therapy does not alter the natural history of retinal degeneration. Proc Natl Acad Sci 110: E1706.

Cong L, Ran FA, Cox D, Lin S, Barretto R, Habib N, Hsu PD, Wu X, Jiang W, Marraffini LA, et al. 2013. Multiplex genome engineering using CRISPR/Cas systems. Science 339: 819-823e.

Conley SM, Naash MI. 2010. Nanoparticles for retinal gene therapy. Prog Retin Eye Res 29: 376-397.

Dalkara D, Byrne LC, Klimczak RR, Visel M, Yin L, Merigan WH, Flannery JG, Schaffer DV. 2013. In vivo-directed evolution of a new adeno-associated virus for therapeutic outer retinal gene delivery from the vitreous. Sci Transl Med 5: $189 \mathrm{ra} 176$.

Kay CN, Ryals RC, Aslanidi GV, Min SH, Ruan Q, Sun J, Dyka FM, Kasuga D, Ayala AE, Van Vliet K, et al. 2013. Targeting photoreceptors via intravitreal delivery using novel, capsid-mutated AAV vectors. PLoS ONE 8: e62097.

Lai CM, Estcourt MJ, Himbeck RP, Lee SY, Yew-San Yeo I, Luu C, Loh BK, Lee MW, Barathi A, Villano J, et al. 2012. Preclinical safety evaluation of subretinal AAV2.sFlt-1 in non-human primates. Gene Ther 19: 999-1009.

Maclachlan TK, Lukason M, Collins M, Munger R, Isenberger E, Rogers C, Malatos S, Dufresne E, Morris J, Calcedo R, et al. 2011. Preclinical safety evaluation of AAV2-sFLT01-A gene therapy for age-related macular degeneration. Mol Ther 19: 326-334.

MacLaren RE, Groppe M, Barnard AR, Cottriall CL, Tolmachova T, Seymour L, Clark KR, During MJ, Cremers FP, Black GC, et al. 2014. Retinal gene therapy in patients with choroideremia: Initial findings from a phase $1 / 2$ clinical trial. Lancet 383: 1129-1137.

Maguire AM, Simonelli F, Pierce EA, Pugh EN Jr, Mingozzi F, Bennicelli J, Banfi S, Marshall KA, Testa F, Surace EM, et al. 2008. Safety and efficacy of gene transfer for Leber's congenital amaurosis. N Engl J Med 358: $2240-$ 2248.

Mali P, Yang L, Esvelt KM, Aach J, Guell M, DiCarlo JE, Norville JE, Church GM. 2013. RNA-guided human genome engineering via Cas9. Science 339: 823-826.

Marlhens F, Bareil C, Griffoin JM, Zrenner E, Amalric P, Eliaou C, Liu SY, Harris E, Redmond TM, Arnaud B, et al. 1997. Mutations in RPE65 cause Leber's congenital amaurosis. Nat Genet 17: 139-141.

O’Reilly M, Kohn DB, Bartlett J, Benson J, Brooks PJ, Byrne BJ, Camozzi C, Cornetta K, Crystal RG, Fong Y, et al. 2013. Gene therapy for rare diseases: Summary of a National Institutes of Health workshop, September 13, 2012. Hum Gene Ther 24: 355-362.

Sahel JA, Roska B. 2013. Gene therapy for blindness. Annu Rev Neurosci 36: 467-488.

Simonato M, Bennett J, Boulis NM, Castro MG, Fink DJ, Goins WF, Gray SJ, Lowenstein PR, Vandenberghe LH, Wilson TJ, et al. 2013. Progress in gene therapy for neurological disorders. Nat Rev Neurol 9: 277-291.

Tolmachova T, Tolmachov OE, Barnard AR, de Silva SR, Lipinski DM, Walker NJ, Maclaren RE, Seabra MC. 2013. Functional expression of Rab escort protein $1 \mathrm{fol}-$ lowing AAV2-mediated gene delivery in the retina of cho- 
roideremia mice and human cells ex vivo. J Mol Med (Berl) 91: 825-837.

Townes-Anderson E. 2013. Increased levels of gene therapy may not be beneficial in retinal disease. Proc Natl Acad Sci 110: E1705.

Vandenberghe LH, Auricchio A. 2012. Novel adeno-associated viral vectors for retinal gene therapy. Gene Ther 19: $162-168$.

Vandenberghe LH, Bell P, Maguire AM, Cearley CN, Xiao R, Calcedo R, Wang L, Castle MJ, Maguire AC, Grant R, et al. 2011. Dosage thresholds for AAV2 and AAV8 pho- toreceptor gene therapy in monkey. Sci Transl Med 3: 88ra54.

Wojno AP, Pierce EA, Bennett J. 2013. Seeing the light. Sci Transl Med 5: 175fs 178.

Zallocchi M, Binley K, Lad Y, Ellis S, Widdowson P, Iqball S, Scripps V, Kelleher M, Loader J, Miskin J, et al. 2014 EIAV-based retinal gene therapy in the shaker1 mouse model for usher syndrome type 1B: Development of UshStat. PLoS ONE 9: e94272.

Zoltick PW, Wilson JM. 2001. Regulated gene expression in gene therapy. Ann NY Acad Sci 953: 53-63. 


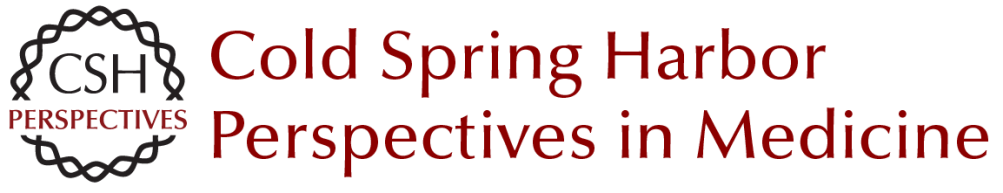

\section{What Is Next for Retinal Gene Therapy?}

Luk H. Vandenberghe

Cold Spring Harb Perspect Med 2015; doi: 10.1101/cshperspect.a017442 originally published online April 15, 2015

\section{Subject Collection Retinal Disorders: Genetic Approaches to Diagnosis and Treatment}

Trial by "Firsts": Clinical Trial Design and Regulatory Considerations in the Development and Approval of the First AAV Gene Therapy Product in the United States

Kathleen Z. Reape and Katherine A. High

Immunology of Retinitis Pigmentosa and Gene Therapy-Associated Uveitis

Paul Yang, Debarshi Mustafi and Kathryn L. Pepple

Developing New Vectors for Retinal Gene Therapy Emilia A. Zin, Bilge E. Ozturk, Deniz Dalkara, et al.

Beyond the NEI-VFQ: Recent Experience in the Development and Utilization of Patient-Reported Outcomes for Inherited Retinal Diseases

Todd Durham, Judit Banhazi, Francesco Patalano, et al.

Electronic Retinal Prostheses

Daniel Palanker

Alternative RNA Splicing in the Retina: Insights and Perspectives

Casey J. Keuthan, Sadik Karma and Donald J. Zack

X-Linked Retinoschisis

Cristy A. Ku, Lisa W. Wei and Paul A. Sieving

A Systematic Review of Optogenetic Vision Restoration: History, Challenges, and New Inventions from Bench to Bedside

Antonia Stefanov and John G. Flannery
Lessons Learned from the Development of the

First FDA-Approved Gene Therapy Drug,

Voretigene Neparvovec-rzyl Jean Bennett and Albert M. Maguire

Therapeutic Gene Editing in Inherited Retinal Disorders Jinjie Ling, Laura A. Jenny, Ashley Zhou, et al.

Cell-Based Therapies: Strategies for Regeneration Marina Pavlou and Thomas A. Reh

The Importance of Natural History Studies in Inherited Retinal Diseases

Allison Ayala, Janet Cheetham, Todd Durham, et al.

Photoreceptor Cell Replacement Using

Pluripotent Stem Cells: Current Knowledge and Remaining Questions

Christelle Monville, Olivier Goureau and Karim Ben M'Barek

iPSC-RPE in Retinal Degeneration: Recent

Advancements and Future Perspectives

Tadao Maeda and Masayo Takahashi

Retinal Degeneration Animal Models in Bardet-

Biedl Syndrome and Related Ciliopathies Clarisse Delvallée and Hélène Dollfus

Mobility Testing and Other Performance-Based

Assessments of Functional Vision in Patients with Inherited Retinal Disease

Daniel Chung, Colas Authié and Laure Blouin

For additional articles in this collection, see http://perspectivesinmedicine.cshlp.org/cgi/collection/ 
For additional articles in this collection, see http://perspectivesinmedicine.cshlp.org/cgi/collection/ 